\title{
REZENSIONEN
}

\section{Das politische System der EU: für Einsteiger und Experten}

\author{
Tömmel, Ingeborg: Das politische System der EU, Oldenbourg Wissenschaftsverlag, München I \\ Wien 2008, 3. vollständig überarbeitete und aktualisierte Auflage, 307 Seiten, € 29,80.
}

Wessels, Wolfgang: Das politische System der Europäischen Union, VS Verlag für Sozialwissenschaften, Wiesbaden 2008, 560 Seiten, €24,90.

Die Europäische Union ist ein kompliziertes Gebilde und mit den herkömmlichen politikwissenschaftlichen Begriffen wie Staat oder internationale Organisation kaum zu fassen. Politikwissenschaftler behelfen sich deshalb gerne mit dem Begriff „sui generis“: Die EU ist eben ein System der eigenen Art. Das macht nun allerdings das Verständnis ihrer Entstehung, Entwicklung und Funktionsweise nicht einfacher. Im Gegenteil: im Laufe ihrer Entwicklung hat die EU nicht nur immer größeren Einfluss auf das Leben der Unionsbürger gewonnen, sie ist auch immer komplizierter und undurchschaubarer geworden. Der Aufgabe, Licht in das Dickicht europäischer Unübersichtlichkeit zu bringen, widmen sich die von Ingeborg Tömmel und Wolfgang Wessels vorgelegten Lehrbücher auf ganz unterschiedliche, aber gleichermaßen erfolgreiche Weise.

Mit der dritten Auflage hat Ingeborg Tömmel eine vollständig überarbeitete und aktualisierte Version ihres bewährten Lehrbuchs vorgelegt, das die Entwicklungen seit der Osterweiterung 2007 und dem Vertrag von Lissabon einbezieht. Im ersten Kapitel entwickelt Tömmel ein Hypothesenset, das dem Band als analytischer Rahmen dient. Darauf aufbauend analysieren die folgenden drei Kapitel die Entwicklung, Struktur, und Funktionsweise der EU im engeren Sinne. Dass die EU mehr ist als nur ihre bekannten vier Institutionen, macht das fünfte Kapitel zur erweiterten Systemstruktur deutlich, das weitere Institutionen auf europäischer Ebene sowie der nationalen wie regionalen Politik- und Verwaltungsebene und nicht-staatliche Akteure einbezieht. Auf dieser Basis diskutiert Tömmel Stärken und Schwächen der EU im Hinblick auf die Effizienz, Effektivität und demokratische Legitimation des Systems. Im letzten Kapitel werden die Argumente zu einem Gesamtbild zusammengeführt. Anhand unterschiedlicher Beschreibungen der EU als Verhandlungs-, Verflechtungs- und Mehrebenensystem wird ihr Staatscharakter analysiert und, in einem zweiten Schritt, die Herausbildung der EU vor allem aus dem systemimmanenten Konflikt zwischen intergouvernementalen und supranationalen Elementen erklärt.

Wie schon seine beiden Vorgänger besticht der Band vor allem durch die anschaulich und flüssig geschriebene, aber präzise Betrachtung der Entwicklungs- und Funktionsdynamiken des politischen Systems der EU. Anstatt eine Fülle von Details zu den formalen Strukturen, Institutionen oder Politikfeldern zu bieten, konzentriert sich Tömmel auch in dieser Auflage auf die politikwissenschaftlich fundierte Analyse der Systemstruktur, der Integrationsdynamik sowie der Funktionslogik der EU. Wünschenswert wäre allein gewesen, die Überarbeitung auch für eine bessere didaktische Aufbereitung zu nutzen. Ein Stichwort- und Personenregister hätten einen leichteren Zugriff ermöglicht, ein etwas großzügigerer Umgang mit Grafiken und Abbildungen für noch mehr Anschaulichkeit gesorgt und weiterführende Hinweise den Einstieg in die umfangreiche EU-Literatur erleichtert. Wer 
ein Nachschlagewerk sucht, ist mit dem Band eher schlecht bedient. Für alle, die eine Einführung benötigen und die EU auch verstehen möchten, ist er hervorragend geeignet.

Detailreichtum, Anschaulichkeit und didaktische Aufbereitung sind hingegen die großen Vorteile des von Wolfgang Wessels vorgelegten Buches. Eine Einführung im ersten Kapitel bietet einen Überblick über die politische und wirtschaftliche Bedeutung der EU und skizziert unterschiedliche wissenschaftliche Ansätze zur Erfassung ihres politischen Systems sowie Theorien zur Erklärung des Integrationsprozesses. In drei umfangreichen Kapiteln werden anschließend die Entwicklung, die wesentlichen Institutionen sowie die Formen des Regierens in unterschiedlichen „Aktivitätsbereichen“ der EU detailreich, aber anschaulich dargestellt. Dabei beschränkt sich Wessels auch keineswegs auf die formalen Strukturen und Prozesse der EU, sondern kontrastiert durchgängig geschriebenen Verfassungstext und gelebte Verfassungswirklichkeit der EU. Gelungen ist auch die umfassende Einbeziehung der Rechtsprechung des Europäischen Gerichtshofs, die dem Leser einen Einblick in eine wichtige, in Lehrbüchern manchmal vernachlässigte Integrationsdynamik ermöglicht. Und obgleich sein erklärtes Hauptziel die Vermittlung von Grundkenntnissen zur EU ist, verharrt Wessels nicht in der Deskription, sondern diskutiert regelmäßig unterschiedliche wissenschaftliche Perspektiven auf Aspekte des Integrationsprozesses, der Institutionen und Entscheidungsverfahren. Im abschließenden Kapitel werden Optionen der kontinuierlichen Vertiefung und Erweiterung der EU ebenso diskutiert wie Szenarien der flexiblen Weiterentwicklung (zum Beispiel „Kerneuropa“ oder „Variable Geometrie“) oder der Rückbildung der EU.

Besonders gelungen ist die didaktische Aufbereitung des Bandes. In jedem Kapitel erleichtern „Eckpunkte“ den Einstieg; Merkpunkte, Fragen zur Wiederholung des Stoffs und Thesen zur Diskussion runden jedes Unterkapitel ab, und weiterführende Literaturhinweise erleichtern die Orientierung im Dschungel der EU-Literatur. Vor allem die Verwendung zahlreicher Photos, Tabellen, Abbildungen und Auszüge aus Originaldokumenten sorgen dafür, dass die EU, ihre Entwicklung, Institutionen und Akteure greifbarer werden. Wessels ist damit ein umfangreiches, gleichzeitig aber interessantes und spannend zu lesendes Kompendium zur EU gelungen, das sich an Einsteiger wie Experten richtet und auf keinem Lehrplan fehlen sollte.

Katrin Auel

Europäische Parteien: umfassende Analysen und spezialisierte Einzelstudie

Mittag, Jürgen (Hrsg.): Politische Parteien und europäische Integration. Entwicklungen und Perspektiven transnationaler Parteienkooperation in Europa, Klartext Verlag, Essen 2006, 810 Seiten, $€ 79$,-.

Huber, Til: Makler der Demokratie - Das Potenzial europäischer Parteien am Beispiel der Sozialdemokratischen Partei Europas (Münchner Beiträge zur europäischen Einigung, Band 19), Nomos Verlagsgesellschaft, Baden-Baden 2007, 124 Seiten, €19,-.

Parteien sind ein wichtiges Element politischer Systeme; ihr tatsächlicher Einfluss hängt jedoch ganz wesentlich davon ab, wie die politische Ordnung konkret beschaffen ist und welche Rolle ihnen im politischen Willensbildungs- und Entscheidungsprozess zukommt. 